\title{
Acceptability and Efficacy of an Emollient Containing Ceramide-Precursor Lipids and Moisturizing Factors for Atopic Dermatitis in Pediatric Patients
}

\author{
Kam Lun Hon · Nga Hin Pong • Shuxin Susan Wang • \\ Vivian W. Lee $\cdot$ Nai Ming Luk $\cdot$ Ting Fan Leung
}

Published online: 2 March 2013

(C) The Author(s) 2013. This article is published with open access at Springerlink.com

\begin{abstract}
Background Atopic eczema or dermatitis (AD) is associated with atopy and is characterized by reduced skin hydration and an impaired skin barrier in the epidermis. We investigated the patient acceptability and efficacy of an emollient containing ceramide-precursor lipids and moisturizing factors (LMF) in AD.

Methods Consecutive AD patients were recruited. Swabs and cultures were obtained from the right antecubital fossa and the worst-affected eczematous area, and disease severity [according to the SCORing Atopic Dermatitis (SCORAD) Index], skin hydration, and transepidermal water loss (TEWL) were measured prior to and after 2 weeks' use of the LMF moisturizer. The general acceptability of treatment was documented as being 'very good', 'good', 'fair', or 'poor'.

Results Twenty-four AD patients [mean age 13.8 (standard deviation 5.7) years] were recruited. Two thirds of the patients reported very good or good acceptability of the LMF moisturizer, whereas one third reported fair or poor acceptability. There were no inter-group differences in the pre-use clinical parameters of age, objective SCORAD score, pruritus score, sleep disturbance score, skin
\end{abstract}

K. L. Hon $(\bowtie) \cdot$ N. H. Pong · S. S. Wang · T. F. Leung Department of Paediatrics, Prince of Wales Hospital,

The Chinese University of Hong Kong, 6/F,

Clinical Science Building, Shatin, Hong Kong

e-mail: ehon@hotmail.com

V. W. Lee

School of Pharmacy, The Chinese University of Hong Kong,

Shatin, Hong Kong

N. M. Luk

Dermatology Research Centre, The Chinese University of Hong

Kong, Shatin, Hong Kong hydration, TEWL, topical corticosteroid use, oral antihistamine use, or acceptability of previously used proprietary emollients. However, patients in the fair/poor acceptability group were more likely to have Staphylococcus aureus colonization and to be female (odds ratio $13,95 \%$ confidence interval $1.7-99.4 ; p=0.021$ ). Following use of the LMF moisturizer, the objective SCORAD score, pruritus score, and sleep disturbance score were lower in the very good/good acceptability group than in the fair/poor acceptability group. The mean objective SCORAD score improved (from 31.5 to $25.7 ; p=0.039$ ) and skin hydration improved [from 30.7 arbitrary units (a.u.) to 36.0 a.u.; $p=0.021]$ in the very good/good acceptability group. When the data were analyzed for the strength of the agreement of the rating of acceptability, the $\kappa$ values were 0.338 (fair) for use of body wash and 0.118 (poor) for use of emollients before and after the trial.

Conclusion The LMF moisturizer was considered acceptable by two thirds of the patients with AD. It seems that patients who found the moisturizer acceptable were less likely to be female or to be colonized by $S$. aureus before switching to the product, and they had less severe eczema, less pruritus, and less sleep disturbance after its use than patients who did not find the product acceptable. Gender and $S$. aureus colonization may have influenced the patient acceptability and clinical efficacy of the LMF moisturizer. The lack of agreement with regard to the acceptability of the moisturizer implies that there is room for parent/patient education to improve compliance.

\section{Introduction}

Atopic eczema or dermatitis (AD) is a chronically relapsing dermatosis associated with atopy and is characterized 
by reduced skin hydration, impaired skin integrity [transepidermal water loss (TEWL)], and poor quality of life as a result of deficient ceramides in the epidermis [1]. Regular application of a moisturizer is the key to management of AD. Moisturizer therapy for AD is significantly complicated by the diversity of disease manifestations and by a variety of complex immune abnormalities [1]. Filaggrin (filament-aggregating protein) has an important function in epidermal differentiation and barrier function, and null mutations within the filaggrin $(F L G)$ gene are major risk factors for developing AD [2-6]. Recent advances in the understanding of the pathophysiological process of $\mathrm{AD}$ have led to the production of new moisturizers and topical skin products containing ceramides, pseudoceramides, or natural moisturizing factors targeted at correcting the reduced amount of ceramides and natural moisturizing factors in the stratum corneum [7]. However, many proprietary products that claim to contain these ingredients have no or only limited studies to document their clinical efficacy. Furthermore, independently of the ingredients, patient preference and acceptability may influence the outcomes of topical treatment [8]. The purposes of this study were to investigate the patient acceptability of a product containing ceramide-precursor lipids and moisturizing factors (LMF), and to evaluate its efficacy in improving the clinical and biophysiological properties of the skin of $\mathrm{AD}$ patients.

\section{Methods}

Patients with $\mathrm{AD}$ were recruited from the pediatric dermatology clinic at a teaching hospital. AD was diagnosed according to the UK Working Party's criteria [9]. Skin hydration, TEWL on the right forearm $(2 \mathrm{~cm}$ below the antecubital flexure), and disease severity [according to the SCORing Atopic Dermatitis (SCORAD) Index] were measured. We have previously described our method of standardizing measurements of skin hydration and TEWL [10]. After acclimatization in the consulting room with the patient sitting comfortably in a chair for 20 to 30 minutes, skin hydration [in arbitrary units (a.u.)] and TEWL (in $\mathrm{g} / \mathrm{m}^{2} / \mathrm{h}$ ) were measured with a Mobile Skin Center ${ }^{\circledR}$ MSC 100 equipped with a Corneometer ${ }^{\circledR}$ CM 825 and a Tewameter $^{\circledR}$ TM 210 probe (Courage \& Khazaka Electronic $\mathrm{GmbH}$, Cologne, Germany), according to the manufacturer's instructions. We documented that a site $2 \mathrm{~cm}$ distal to the right antecubital flexure was optimal for standardization. Oozing and infected areas were avoided by moving the probe slightly sideways [10]. The clinical severity of AD was assessed with the SCORAD Index [11, 12].

Patients were given a liberal supply of the LMF moisturizer $\left(\right.$ Cetaphil ${ }^{\circledR}$ RESTORADERM ${ }^{\text {TM }}$ Lotion; Galderma
Canada Inc., Thornhill, ON, Canada) and moisturizing wash $\left(\right.$ Cetaphil $^{\circledR}$ RESTORADERM $^{\mathrm{TM}}$ Wash; Galderma Canada Inc.). The moisturizer claims to contain purified water, glycerin, caprylic/capric triglyceride, Helianthus annuus (sunflower) seed oil, pentylene glycol, Butyrospermum parkii (shea butter), sorbitol, cyclopentasiloxane, cetearyl alcohol, behenyl alcohol, glyceryl stearate, tocopheryl acetate, hydroxypalmitoyl sphinganine $(0.01 \%$ $\mathrm{w} / \mathrm{w})$, cetyl alcohol, arginine $(0.50 \% \mathrm{w} / \mathrm{w})$, disodium ethylene dicocamide polyethylene glycol (PEG)-15 disulfate, glyceryl stearate citrate, niacinamide, sodium pyrrolidone carboxylate (PCA) $[0.50 \% \mathrm{w} / \mathrm{w}]$, ceteareth-20, sodium polyacrylate, caprylyl glycol, allantoin, citric acid, panthenol, dimethiconol, disodium ethylenediaminetetraacetic acid (EDTA), and sodium hyaluronate. Hydroxypalmitoyl sphinganine is a ceramide precursor. Arginine and sodium PCA are natural moisturizing factors. Arginine acts as a substrate not only for arginase but also for nitric oxide synthase.

The moisturizing wash contains purified water, B. parkii, sodium trideceth sulfate, glycerin, H. annuиs seed oil, sodium chloride, sodium lauramphoacetate, cocamide monoethanolamine (MEA), citric acid, niacinamide, sodium PCA $(0.50 \%$ w/w), tocopheryl acetate, 1,2-hexanediol and caprylyl glycol, disodium EDTA, guar hydroxypropyltrimonium chloride, allantoin, potassium sorbate, arginine $(0.10 \% \mathrm{w} / \mathrm{w})$, and methylisothiazolinone.

The patients were instructed not to use any other topical treatment except for their usual corticosteroid on an asnecessary basis. They were encouraged to use the LMF moisturizer at least twice daily on the flexures and areas with eczema. In the event that the emollient effect was not satisfactory, they could use their usual emollient and medications, but the frequency of such use was to be reported, and they must continue using the LMF moisturizer. The patients were reviewed at the end of 2 weeks. Measurements of the SCORAD score, Children's Dermatology Life Quality Index (CDLQI), skin hydration, and TEWL were repeated. The patient's global or general acceptability of treatment (GAT) was recorded as 'very good', 'good', 'fair', or 'poor' [8, 13]. Ethical approval for the study was obtained from the Clinical Research Ethics Committee of the Chinese University of Hong Kong, and written informed consent was obtained from each patient and his/her guardian.

Continuous data are expressed as means and standard deviations (SDs). The Mann-Whitney U test for inter-group comparisons and the McNemar test for within-group comparisons with small numbers of subjects were used. Categorical data are presented as counts. The $\chi^{2}$ test or Fisher's exact test, where appropriate, were used to compare categorical data. $\kappa$ values were determined for the previously used proprietary products and for the LMF 
moisturizer and moisturizing wash. All comparisons were two-tailed, and $p$ values of $\leq 0.05$ were considered statistically significant.

\section{Results}

Between December 2011 and June 2012, 24 patients [63\% male; mean age 13.8 (SD 5.7) years] with AD were recruited and treated with applications of the LMF moisturizer and moisturizing wash. Compliance was good, and patients generally managed to use the moisturizer daily. Two thirds reported very good or good acceptability of the LMF moisturizer, whereas one third reported fair or poor acceptability (Tables 1, 2; the male percentages were $81 \%$ and $25 \%$, respectively; $p=0.021$ ).

There were no inter-group differences in pre-use clinical parameters of age, the objective SCORAD score, pruritus score, sleep disturbance score, skin hydration, TEWL, topical corticosteroid use, oral antihistamine use, or acceptability of the previously used proprietary emollients. However, patients in the fair/poor acceptability group were more likely to have Staphylococcus aureus colonization and to be female (odds ratio 13, $95 \%$ confidence interval $1.7-99.4 ; p=0.021)$. Following use of the LMF moisturizer, the objective SCORAD score, pruritus score, and sleep disturbance scores were lower in the very good/good acceptability group than in the fair/poor acceptability group. The mean objective SCORAD score improved (from $31.5 \mathrm{~g} / \mathrm{m}^{2} / \mathrm{h}$ to $25.7 \mathrm{~g} / \mathrm{m}^{2} / \mathrm{h} ; p=0.039$ ) and skin hydration improved (from 30.7 a.u. to 36.0 a.u.; $p=0.021$ ) in the very good/good acceptability group.

When the data were analyzed for the strength of the agreement of the rating of acceptability, the $\kappa$ values were 0.338 (fair) for use of body wash and 0.118 (poor) for use of emollients before and after the trial. Neither result reached statistical significance, implying that there appeared to be no consistency in agreement (or preference). Patients who preferred the LMF moisturizer or moisturizing wash may or may not have come from the group of poor/fair acceptability of their previous emollient or body wash. Previously used products included emulsifying ointment, $\mathrm{QV}^{\mathrm{TM}}$, Johnson and Johnson, Sebamed ${ }^{\circledR}$, and various other proprietary products.

\section{Discussion}

$\mathrm{AD}$ is a chronically relapsing dermatosis characterized by pruritus, erythema, vesiculation, papulation, exudation, excoriation, crusting, scaling, and sometimes lichenification $[1,14]$. Secondary bacterial infection, most commonly with $S$. aureus, is the main complication $[1,15,16]$. In the brickand-mortar hypothesis, the stratum corneum (the outermost layer of the epidermis) normally consists of fully differentiated corneocytes surrounded by a lipid-rich matrix containing cholesterol, free fatty acids, and ceramides. In AD, lipid metabolism is abnormal, causing a deficiency of ceramides and natural moisturizing factors, and impairment of epidermal barrier function, which leads to increased TEWL $[1,7,17,18]$. It has been shown that loss-of-function mutations in the FLG gene predispose to AD [2-6, 19, 20]. The protein is present in the granular layers of the epidermis. The keratohyalin granules in the granular layers are predominantly composed of profilaggrin [21]. Filaggrin aggregates the keratin cytoskeleton system to form a dense protein-lipid matrix, which is cross-linked by transglutaminases to form a cornified cell envelope [4, 21]. The latter prevents epidermal water loss and impedes the entry of allergens, infectious agents, and chemicals [4, 22].

The key to management of AD and dry skin conditions, especially in between episodes of flare ups, is frequent use of an appropriate moisturizer [1]. Hydration of the skin helps to improve dryness, reduce pruritus, and restore the disturbed skin's barrier function. Bathing without use of a moisturizer may compromise skin hydration [23-25]. Hence, use of emollients is of paramount importance in both prevention and management of $\operatorname{AD}[1,20]$.

Many proprietary emollients claim to replace ceramide ingredients, but few have been tested. This pilot study

Table 1 Global acceptability of treatment ${ }^{\mathrm{a}}$

\begin{tabular}{lcccc}
\hline Acceptability & \multicolumn{2}{l}{ Emollient $[n]$} & & \multicolumn{2}{c}{ Body wash $[n]$} \\
\cline { 2 - 5 } & LMF moisturizer & Other proprietary product & & Moisturizing wash \\
\hline Very good & 3 & 1 & 3 & 0 \\
Good & 13 & 13 & 10 & 11 \\
Fair & 7 & 10 & 0 & 13 \\
Poor & 1 & 0 & 0 \\
\hline
\end{tabular}

$L M F$ ceramide-precursor lipids and moisturizing factors

a When the data were analyzed for the strength of the agreement of the rating of acceptability, the $\kappa$ values were 0.338 (fair) for use of body wash and 0.118 (poor) for use of emollients before and after the trial 
Table 2 Acceptability and efficacy of treatment with the LMF moisturizer ${ }^{\mathrm{a}}$

\begin{tabular}{|c|c|c|c|c|c|c|c|c|}
\hline \multirow[t]{2}{*}{ Parameter } & \multicolumn{2}{|c|}{$\begin{array}{l}\text { Very good/good } \\
\text { acceptability }(n=16)\end{array}$} & \multicolumn{2}{|c|}{$\begin{array}{l}\text { Fair/poor acceptability } \\
(n=8)\end{array}$} & \multicolumn{4}{|l|}{$p$ values ${ }^{\mathrm{b}}$} \\
\hline & $\begin{array}{l}\text { (1) Pre- } \\
\text { treatment }\end{array}$ & $\begin{array}{l}\text { (2) Post- } \\
\text { treatment }\end{array}$ & $\begin{array}{l}\text { (3) Pre- } \\
\text { treatment }\end{array}$ & $\begin{array}{l}\text { (4) Post- } \\
\text { treatment }\end{array}$ & $\begin{array}{l}\text { (1) } \\
\text { versus (2) }\end{array}$ & $\begin{array}{l}(3) \\
\text { versus (4) }\end{array}$ & $\begin{array}{l}\text { (1) } \\
\text { versus (3) }\end{array}$ & $\begin{array}{l}(2) \\
\text { versus (4) }\end{array}$ \\
\hline \multicolumn{9}{|l|}{ Global acceptability of treatment } \\
\hline Male gender $[\mathrm{n}(\%)]$ & & $13(81)^{\mathrm{c}}$ & & $2(25)$ & & & & 0.021 \\
\hline Age [years] & & $13.2(5.7)$ & & $14.8(5.8)$ & & & & 0.70 \\
\hline Objective SCORAD score (SD) & $31.5(13.7)$ & $25.7(14.0)$ & $42.3(22.2)$ & $40.5(17.6)$ & 0.039 & 0.46 & 0.086 & 0.035 \\
\hline Pruritus score (SD) & $5.6(1.8)$ & $4.9(2.0)$ & $6.5(2.3)$ & $6.6(2.1)$ & 0.20 & 0.98 & 0.35 & 0.043 \\
\hline Sleep disturbance score (SD) & $3.6(3.1)$ & $3.3(2.6)$ & $5.8(3.3)$ & $6.3(3.2)$ & 0.36 & 0.63 & 0.15 & 0.032 \\
\hline Skin hydration [a.u. (SD)] & $30.7(12.3)$ & $36.0(10.5)$ & $36.1(16.0)$ & $39.2(19.8)$ & 0.021 & 0.38 & 0.36 & 0.74 \\
\hline TEWL $\left[\mathrm{g} / \mathrm{m}^{2} / \mathrm{h}(\mathrm{SD})\right]$ & $11.5(3.4)$ & $12.3(4.4)$ & $11.8(2.8)$ & $12.0(2.0)$ & 0.56 & 0.64 & 0.76 & 0.39 \\
\hline $\begin{array}{l}\text { Staphylococcus aureus in the antecubital } \\
\text { fossa }[n]\end{array}$ & 6 & 7 & 6 & 6 & 1.0 & 1.0 & 0.19 & 0.21 \\
\hline Worst-affected eczematous area & 8 & 10 & 8 & 8 & 0.63 & NA & 0.022 & 0.066 \\
\hline Topical corticosteroid use $[n]$ & 8 & 6 & 5 & 3 & 0.50 & 0.50 & 0.68 & 1.0 \\
\hline Antihistamine use $[n]$ & 5 & 3 & 6 & 4 & 0.50 & 0.50 & 0.082 & 0.17 \\
\hline
\end{tabular}

a.u. arbitrary units, $L M F$ ceramide-precursor lipids and moisturizing factors, NA not applicable, SCORAD SCORing atopic dermatitis, $S D$ standard deviation, TEWL transepidermal water loss

a Values are expressed as means (SDs) unless stated otherwise

${ }^{\mathrm{b}} p$ values of $\leq 0.05$ are statistically significant. The $\mathrm{p}$ values presented are for comparisons between pre- and post-treatment in the very good/ good acceptability group [(1) versus (2)], comparisons between pre- and post-treatment in the fair/poor acceptability group [(3) versus (4)], comparisons between the very good/good and fair/poor acceptability groups pre-treatment [(1) versus (3)], and comparisons between the very good/good and fair/poor acceptability groups post-treatment [(2) versus (4)]

c The odds ratio for very good/good acceptability of LMF moisturizer in female patients was 0.089 (95\% confidence interval $0.006-0.793$ )

explored patient acceptability of a moisturizer containing lipids and natural moisturizing factors, and evaluated its efficacy in AD. We showed that the LMF moisturizer was considered acceptable by two thirds of the patients with AD. It seems that patients who found the moisturizer acceptable were less likely to be female or to be colonized by $S$. aureus before switching to the LMF moisturizer, and they had less severe eczema, less pruritus, and less sleep disturbance following its use than patients who did not find the product acceptable. Gender and S. aureus colonization may have influenced the patient acceptability and clinical efficacy of the LMF moisturizer. In the wider context, AD is a complex multifactorial atopic disease, and monotherapy targeted merely at replacement of ceramides, pseudoceramides, or filaggrin degradation products at the epidermis is often suboptimal. In particular, colonization with $S$. aureus must be adequately treated before emollient treatment can be optimized [16].

Despite claims about their efficacy, little evidence has demonstrated short- or long-term usefulness of many proprietary products. Some ceramides and pseudoceramides have been studied and added to commercial moisturizers to mimic natural skin-moisturizing factors, and to influence both TEWL and expression of antimicrobial peptides in patients with $\mathrm{AD}$ [26]. Chamlin et al. [5] assessed the efficacy of a ceramide-dominant, physiological lipid-based emollient, when substituted for currently used moisturizers, in 24 children who were also receiving standard therapy for 'stubborn-to-recalcitrant' AD. All subjects continued their previous therapy (e.g., topical tacrolimus or corticosteroids), only substituting the barrier-repair emollient for their previous moisturizer. Follow-up SCORAD scores improved significantly in 22 of 24 patients by 3 weeks, with further progressive improvement in all patients between 6 and 20 or 21 weeks. TEWL, which was elevated over the involved and uninvolved areas at study entry, decreased in parallel with SCORAD scores and continued to decline even after the SCORAD scores plateaued. Stratum corneum integrity and hydration also improved significantly during therapy. The ultrastructure of the stratum corneum following treatment with the ceramidedominant emollient revealed extracellular lamellar membranes, which were largely absent in baseline stratum corneum samples. The authors concluded that a ceramidedominant barrier-repair emollient represents a safe and useful adjunct to the treatment of childhood AD. 
EpiCeram ${ }^{\circledR}$ consists of a specific combination of ceramides, cholesterol, and fatty acids (in the ratio of $3: 1: 1$ ), which mimic those naturally found in the skin $[27,28]$. Recent studies have shown that EpiCeram ${ }^{\circledR}$ has efficacy similar to that of a mid-potency topical corticosteroid but has a more favorable safety profile [27, 28]. However, those studies did not report objective measurements to demonstrate the efficacy of treatment.

Hon et al. [29] studied skin hydration and TEWL on the forearm and determined the SCORAD score, Nottingham Eczema Severity Score (NESS), CDLQI, and amounts of emollient and cleanser used over a 2-week period in consecutive new patients seen at the pediatric skin clinic. Patients with AD had significantly greater TEWL and less skin hydration at the studied sites. Although both skin dryness and skin hydration were improved, there was no significant improvement in the SCORAD score or TEWL after 2 weeks. In terms of GAT, three quarters of patients with AD and controls rated the combination of the cream and cleanser as good or very good. The authors concluded that liberal use of emollients and bathing cleanser alone does not seem to alter disease severity or TEWL within 2 weeks, implying that additional treatments are necessary to manage AD [29].

In another study, Hon et al. [13] recruited 33 patients with AD to study the clinical and biophysiological effects of twice-daily application of a pseudoceramide-containing cream. Four weeks after the patients started using the pseudoceramide cream, their skin hydration had improved significantly. There was no deterioration in TEWL, eczema severity, or quality of life in these patients. The pseudoceramide cream improved skin hydration but not eczema severity or quality of life over 4 weeks of use $[13,30]$.

A number of topical preparations are available on the market. The actual ingredients of most of these products are a commercial secret of the individual pharmaceutical companies. However, the active ingredients are identified on the packaging. The type of moisturizer or emollient should be tailored to the individual skin condition as well as the child's needs and preferences [31, 32].

In terms of GAT, the current study showed that only two thirds of the AD patients considered the acceptability of the product to be very good or good, and one third considered its acceptability to be fair or poor. It seems that patients who found the LMF moisturizer acceptable were less likely to be female, to be colonized by S. aureus, and to have been using an antihistamine before switching to the moisturizer, and they had less severe eczema and less sleep disturbance following its use than patients who did not find the product acceptable. Gender and $S$. aureus colonization may have influenced the patient acceptability and clinical efficacy of the trial moisturizer. The low acceptability of these products reflects the fact that there is no user consistency in the preference, acceptability, and choice of emollients. The major hindrance to the efficacy of a moisturizer is the patient's perception as to what an ideal moisturizer should be like [8]. This perception varies from person to person. Therefore, the physician caring for a patient with $\mathrm{AD}$ must educate and guide the parents and the patient to choose the most acceptable formulation to ensure optimal compliance. Ultimately, an 'ideal' emollient is an individualized choice that the patient will accept and use.

This pilot study provides insights for further research into ceramide-containing emollients. First, patient acceptance of the strengths, types, and formulations of ceramides and related products needs to be studied in randomized controlled trials of any novel products. Second, efficacy studies holistically focusing on all clinical parameters (namely severity scores, quality-of-life indices, skin hydration, TEWL, S. aureus colonization, and patient acceptability) must be performed. Third, as AD is not a simple epidermal skin disease but, rather, is a complex atopic disease, use of an emollient alone is bound to be suboptimal in efficacy. In the current study, it was evident that $S$. aureus colonization was prevalent especially in patients with moderate-to-severe disease, thus future randomized controlled trials should include a run-in period to eradicate such colonization in order to evaluate the net effects due to the emollient.

\section{Conclusion}

The incorporation of ingredients containing ceramides, pseudoceramides, and natural moisturizing factors into therapeutic moisturizers targets the pathophysiology of AD. Well designed, large-scale, randomized, placebocontrolled trials are needed to document therapeutic effects on disease severity, dermatological biophysical parameters, quality of life, and patient acceptability. Patient acceptability of a certain product is pivotal to compliance and clinical outcome.

Acknowledgments The authors thank Galderma Hong Kong Limited for freely supplying the studied materials. However, the company was not involved in any financial sponsorship, design, or analysis of the research data in this project. Furthermore, no sources of funding were used to conduct the study or to prepare this manuscript.

Conflicts of Interest Drs. Hon and Leung have performed research on eczema therapeutics, and have written about the subject matters of filaggrin and ceramides. Vivian Lee has received an educational grant from AstraZeneca and has had contracts for research with Roche. The authors have no other conflicts of interest that are directly relevant to the content of this article.

Open Access This article is distributed under the terms of the Creative Commons Attribution Noncommercial License which permits any noncommercial use, distribution, and reproduction in any medium, provided the original author(s) and the source are credited. 


\section{References}

1. Leung AK, Hon KL, Robson WL. Atopic dermatitis. Adv Pediatr. 2007;54:241-73.

2. Sandilands A, Terron-Kwiatkowski A, Hull PR, O'Regan GM, Clayton TH, Watson RM, et al. Comprehensive analysis of the gene encoding filaggrin uncovers prevalent and rare mutations in ichthyosis vulgaris and atopic eczema. Nat Genet. 2007;39(5):650-4.

3. Sandilands A, Smith FJ, Irvine AD, McLean WH. Filaggrin's fuller figure: a glimpse into the genetic architecture of atopic dermatitis. J Invest Dermatol. 2007;127:1282-4.

4. Enomoto H, Hirata K, Otsuka K, Kawai T, Takahashi T, Hirota T, et al. Filaggrin null mutations are associated with atopic dermatitis and elevated levels of $\operatorname{IgE}$ in the Japanese population: a family and case-control study. J Hum Genet. 2008;53(7):615-21.

5. Chamlin SL, Kao J, Frieden IJ, Sheu MY, Fowler AJ, Fluhr JW, et al. Ceramide-dominant barrier repair lipids alleviate childhood atopic dermatitis: changes in barrier function provide a sensitive indicator of disease activity. J Am Acad Dermatol. 2002;47(2): 198-208.

6. Maintz L, Novak N. Getting more and more complex: the pathophysiology of atopic eczema. Eur J Dermatol. 2007;17(4):267-83.

7. Hon KL, Leung AKC. Use of ceramides and related products for childhood-onset eczema. Recent Pat Inflamm Allergy Drug Discov. 2013;7(1):12-9.

8. Hon KL, Wang SS, Pong NH, Leung TF. The ideal moisturizer: a survey of parental expectations and practice in childhood-onset eczema. J Dermatol Treat. 2013;24(1):7-12.

9. Williams HC, Burney PG, Pembroke AC, Hay RJ. The UK Working Party's diagnostic criteria for atopic dermatitis: III. independent hospital validation. Br J Dermatol. 1994;131(3):406-16.

10. Hon KL, Wong KY, Leung TF, Chow CM, Ng PC. Comparison of skin hydration evaluation sites and correlations among skin hydration, transepidermal water loss, SCORAD Index, Nottingham Eczema Severity Score, and quality of life in patients with atopic dermatitis. Am J Clin Dermatol. 2008;9(1):45-50.

11. [No authors listed.] Severity scoring of atopic dermatitis: the SCORAD Index. Consensus report of the European Task Force on Atopic Dermatitis. Dermatology 1993;186(1):23-31.

12. Kunz B, Oranje AP, Labreze L, Stalder JF, Ring J, Taieb A. Clinical validation and guidelines for the SCORAD Index: consensus report of the European Task Force on Atopic Dermatitis. Dermatology. 1997;195(1):10-9.

13. Hon KL, Wang SS, Lau Z, Lee HC, Lee KK, Leung TF, et al. Pseudoceramide for childhood eczema: does it work? Hong Kong Med J. 2011;17(2):132-6.

14. Leung DY, Boguniewicz M, Howell MD, Nomura I, Hamid QA. New insights into atopic dermatitis. J Clin Invest. 2004;113(5): 651-7.

15. Hon KL, Lam MC, Leung TF, Kam WY, Li MC, Ip M, et al. Clinical features associated with nasal Staphylococcus aureus colonisation in Chinese children with moderate-to-severe atopic dermatitis. Ann Acad Med Singap. 2005;34(10):602-5.

16. Hon KL, Wang SS, Lee KK, Lee VW, Fan LT, Ip M. Combined antibiotic/corticosteroid cream in the empirical treatment of moderate to severe eczema: friend or foe? J Drugs Dermatol. 2012;11(7):861-4.

17. Hanifin JM, Rajka G. Diagnostic features of atopic dermatitis. Acta Derm Venereol (Stockh). 1980;2:44-7.

18. Hanifin JM. Atopic dermatitis. J Am Acad Dermatol. 1982;6(1):1-13.

19. Palmer CN, Irvine AD, Terron-Kwiatkowski A, Zhao Y, Liao H, Lee SP, et al. Common loss-of-function variants of the epidermal barrier protein filaggrin are a major predisposing factor for atopic dermatitis. Nat Genet. 2006;38(4):441-6.

20. Krakowski AC, Eichenfield LF, Dohil MA. Management of atopic dermatitis in the pediatric population. Pediatrics. 2008;122(4):812-24.

21. Candi E, Schmidt R, Melino G. The cornified envelope: a model of cell death in the skin. Nat Rev Mol Cell Biol. 2005;6(4):328-40.

22. Leung DY, Nicklas RA, Li JT, Bernstein IL, Blessing-Moore J, Boguniewicz M, et al. Disease management of atopic dermatitis: an updated practice parameter. Joint Task Force on Practice Parameters. Ann Allergy Asthma Immunol. 2004;93(3 Suppl 2):S1-21.

23. Lancaster W. Atopic eczema in infants and children. Community Pract. 2009;82(7):36-7.

24. Tarr A, Iheanacho I. Should we use bath emollients for atopic eczema? BMJ. 2009;339:b4273.

25. Hon KL, Leung TF, Wong Y, Li A, Fok TF, et al. A survey of bathing and showering practices in children with atopic eczema. Clin Exp Dermatol. 2005;30(4):351-4.

26. Park KY, Kim DH, Jeong MS, Li K, Seo SJ. Changes of antimicrobial peptides and transepidermal water loss after topical application of tacrolimus and ceramide-dominant emollient in patients with atopic dermatitis. J Korean Med Sci. 2010;25(5): 766-71.

27. Draelos ZD. The effect of ceramide-containing skin care products on eczema resolution duration. Cutis. 2008;81(1):87-91.

28. Madaan A. EpiCeram for the treatment of atopic dermatitis. Drugs Today. 2008;44(10):751-5.

29. Hon KL, Ching GK, Leung TF, Choi CY, Lee KK, Ng PC. Estimating emollient usage in patients with eczema. Clin Exp Dermatol. 2010;35(1):22-6.

30. Kim HJ, Park HJ, Yun JN, Jeong SK, Ahn SK, Lee SH. Pseudoceramide-containing physiological lipid mixture reduces adverse effects of topical steroids. Allergy Asthma Immunol Res. 2011;3(2):96-102.

31. Roos TC, Geuer S, Roos S, Brost H. Recent advances in treatment strategies for atopic dermatitis. Drugs. 2004;64(23):2639-66.

32. Baumer JH. Atopic eczema in children, NICE. Arch Dis Child Educ Pract Ed. 2008;93(3):93-7. 